\title{
APLICACIÓN DE LA CITOMETRÍA DE FLUJO EN VETERINARIA
}

\section{APPLICATION OF FLOW CITOMETRY IN VETERINARY}

\section{Alejandro Córdova Izquierdo ${ }^{1}$, Adrían Emmanuel Iglesias Reyes ${ }^{1}$, Román Espinosa} Cervantes $^{1}$, Juan Eulogio Guerra Liera ${ }^{2}$, Jorge Fabio Inzunza Castro² ${ }^{2}$ Ma. de Lourdes Juárez Mosqueda ${ }^{3}$, Armando Gómez Vázquez ${ }^{4}$ y Blanca Estela Rodriguez Denis ${ }^{5}$

${ }^{1}$ Departamento de Producción Agrícola y Animal. Universidad Autónoma Metropolitana Unidad Xochimilco, México, D.F. ${ }^{2}$ Facultad de Agronomía. Universidad Autónoma de Sinaloa, México. ${ }^{3}$ Departamento de Morfología. FMVZ-UNAM. ${ }^{4}$ División Académica de Ciencias Agropecuarias. Universidad Juárez Autónoma de Tabasco, México. ${ }^{5}$ Práctica privada. Correspondencia con autor: acordova@correo.xoc.uam.mx.

\section{RESUMEN}

La citometría de flujo es una herramienta de análisis de laboratorio que se empezó a usar a principios de los años 50’s con el fin de contar y analizar células sanguíneas, durante la década de los 80`s se convirtió en una técnica cuantitativa apreciada en el área de Ciencias de la Salud y en la actualidad es usada para distintos fines en el campo de la medicina, microbiología, bioquímica, en el laboratorio, para temas ambientales o biológicos, en el campo de la investigación tanto teórica como práctica, que a pesar de no ser muy accesible, permite de una forma rápida, objetiva y cuantitativa analizar células, núcleos, cromosomas, mitocondrias u otras partículas. El uso de la citometría de Flujo (CF) en Veterinaria es relativamente reciente; no obstante, resulta ser un método prometedor. En este trabajo, se hace una breve descripción de los beneficios de la aplicación de la CF, áreas de aplicación, beneficios en veterinaria en general y en particular en la reproducción animal.

PALABRAS CLAVE: Citometría de flujo, medicina veterinaria, reproducción animal, semen. 


\begin{abstract}
Flow citometry is a laboratory analysis tool that began to be used in the early 1950's in order to count and analyze blood cells. During the 1980s it became a quantitative technique appreciated in The area of Health Sciences and is currently used for various purposes in the field of medicine, microbiology, biochemistry, in the laboratory, for environmental or biological issues, in the field of theoretical and practical research, Although not very accessible, it allows a quick, objective and quantitative analysis of cells, nuclei, chromosomes, mitochondria or other particles. The use of Flow Citometry (CF) in Veterinary Medicine is relatively recent; However, proves to be a promising method. In this paper, a brief description of the benefits of application of FC, areas of application, benefits in veterinary in general and in particular in animal reproduction is made.
\end{abstract}

KEY WORDS: Flow citometry, veterinary medicine, animal reproduction, semen.

\title{
INTRODUCCIÓN
}

Citometría es un término genérico que se aplica a cualquier tecnología que es usada para la medición, recuento, comparación u otra caracterización de células. Después de su desarrollo en los años 50, donde se comenzó a usar con el fin de contar y analizar células sanguíneas; después durante la década de los años 80, desde la primera generación de los citómetros de flujo, la citometría de convirtió en una técnica cuantitativa apreciada, de baja complejidad, económica y fácil de mantener en el laboratorio. Dada la evolución en la química de los reactivos y la modernidad de los instrumentos, desde ese momento han habido grandes avances, los citómetros actuales combinan una mezcla de tecnologías modernas tales como: mecánica de fluidos, rayos laser, óptica, electrónica análoga y digital y software computarizados (Loureiro, 2009; Laguado, 2007; Piedras, 2006). La CF representa un método rápido, objetivo y cuantitativo de análisis de células, núcleos, cromosomas, mitocondrias u otras partículas en suspensión. Una de las características analíticas más importantes de los citómetros de flujo es su capacidad de medir múltiples parámetros celulares, como el tamaño, forma, estructura, contenido (análisis multiparámetro) y complejidad de propiedades celulares en líquidos corporales, así como de cualquier partícula tan pequeña como $0.1 \mu \mathrm{m}$ y, por supuesto, cualquier componente celular o función que pueda ser marcada con fluorocromo (Piedras, 2006; Barrera et al., 2004). 
La CF nos indica tres cosas fundamentales sobre una muestra de un experimento: el tamaño de las partículas o células a los cual se le denomina Forward Scatter o FSC, la complejidad relativa interna o granularidad y la intensidad de la fluorescencia de manera relativa (cuando la muestras ha sido teñida con anticuerpos marcados con fluorocromos o con algún tipo de molécula fluorecente) indicando marcadores en la célula contra los cuales los anticuerpos son usados o los fluorocromos han sido dirigidos (Laguado, 2007).

En este trabajo, se hace una breve descripción de los beneficios de la aplicación de la CF, áreas de aplicación, beneficios en veterinaria en general y en particular en la reproducción animal.

\section{BENEFICIOS DE LA APLICACIÓN DE LA CITOMETRÍA DE FLUJO}

El principio en el que se basa esta tecnología es simple: hacer pasar células u otras partículas en suspensión alineadas de una en una, por delante de un haz luminoso. Esta medición en flujo es afectada a alta velocidad (e.g., análisis de 100-1000 partículas por segundo) y garantiza un análisis objetivo de las partículas que constituyen la población de interés. La información producida puede agruparse en dos tipos fundamentales: la generada por dispersión de la luz y la relacionada con la emisión de luz por los fluorocromos presentes en la célula o particular al ser excitados por el rayo luminoso. Las señales luminosas detectadas se transforman en impulsos eléctricos que se amplifican y se convierten en señales digitales que son procesadas en una computadora. Los análisis posteriores de las poblaciones y subpoblaciones se producen mediante el uso de programas de cómputo especializados lo cual permite obtener información valiosa para el investigador o el clínico (fig. 1) (Loureiro, 2009; Laguado, 2007; Barrera et al., 2004). Debido a su capacidad de recolectar información de miles o millones de células al mismo tiempo, la citometría de flujo facilita el análisis multiparametrico como lo realizan otras técnicas de laboratorio. Esta característica de analizar millones de células también facilita la obtención de datos estadísticamente significativos en un periodo de tiempo corto. Este proceso físico-químico que ocurre con la muestra se logra por intermedio de los tres componentes principales de un citómetro de flujo (Tabla 1) el cual es un instrumento muy reconocido en la práctica clínica de rutina pero que ahora ha incrementado su uso en otras aplicaciones no clínicas (Laguado, 2007). 
Tabla 1. Principales sistemas y componentes de un citómetro de flujo.

\begin{tabular}{ll}
\hline SISTEMA & FUNCIÓN \\
\hline Fluidos & Transportar la muestra hacia el haz de luz del láser y \\
& mantener sus propiedades fisicoquímicas. \\
Óptico & Iluminar las partículas de la muestra y obtener las señales \\
& de luz resultantes en los filtros y detectores apropiados \\
Electrónica & Convertir las señales de luz en señales electrónicas que \\
& un computador y programa de cómputo puedan interpretar. \\
\hline
\end{tabular}

Modificado de: Laguado, 2007.

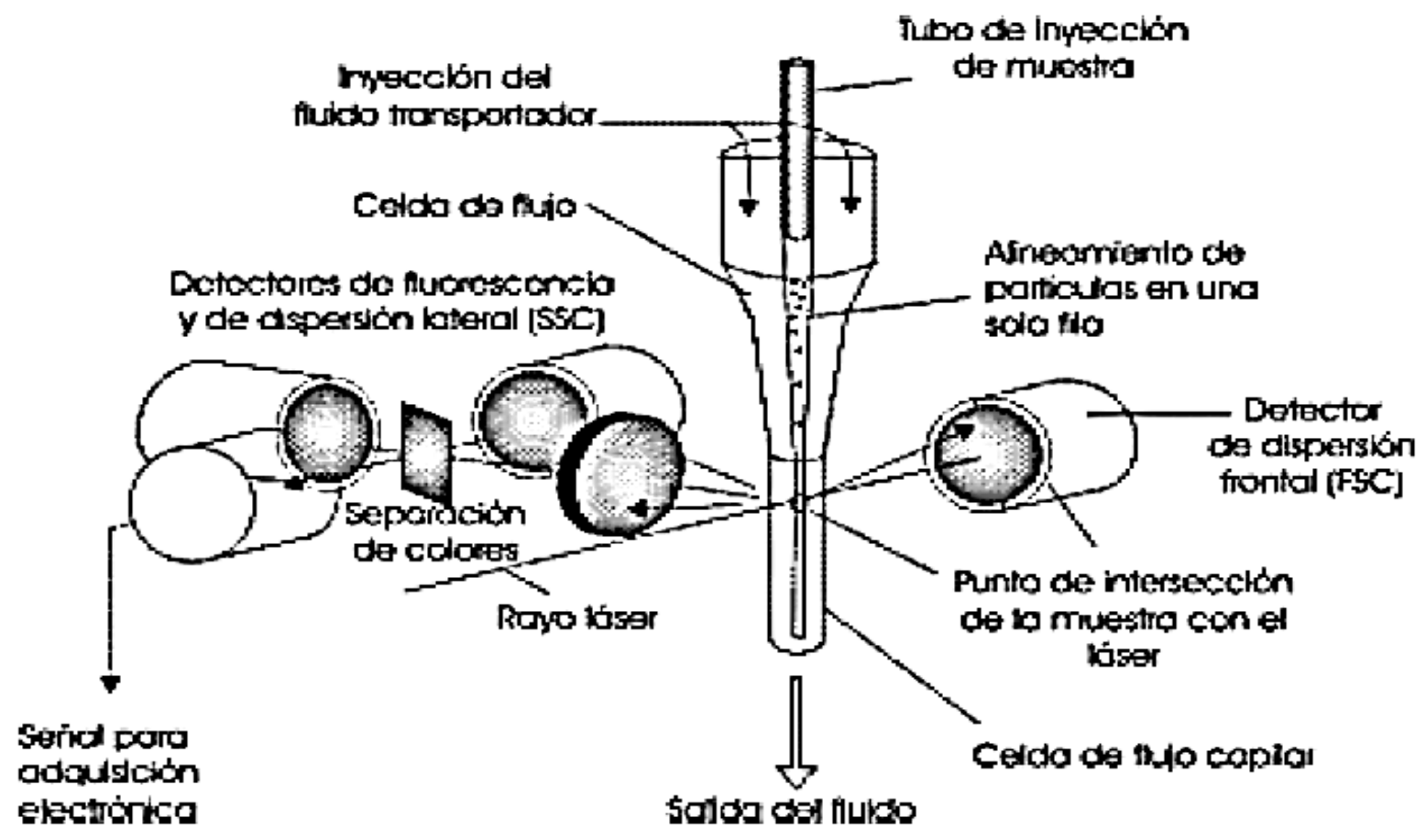

Fig 1. Principio general de citometría de flujo. Modificado de: Barrera et al., 2004

El uso de compuestos fluorescentes denominados fluorocromos, en citometría de flujo ha sido fundamental en el desarrollo de esta técnica. Es muy importante tener en cuenta que cuando se utilizan varios fluorocromos los espectros de emisión deben tener un solapamiento mínimo con objeto de que pueda cuantificarse de forma separada (Fig. 2).

El primer fluorocromo utilizado para el marcaje fue el FITC, que es una pequeña molécula, con una importante afinidad a las proteínas, se excita en azul. Sin embargo para estudios más específicos como es el caso de ácidos nucleicos, los fluorocromos más empleados son el yoduro de propidio (YP), el bromuro de etidio, naranja de acridina, Hoechst 33342, 
naranja de tiazol, la mitramicina y la cromomicina A3; siendo el yoduro de propidio y el bromuro de etidio los más utilizados en citometría de flujo, los cuales se excitan a longitudes de onda de $488 \mathrm{~nm}$ y se unen de forma estequiométrica al ADN de doble cadena, donde se intercalan entre la doble hélice (Matas, 1997).

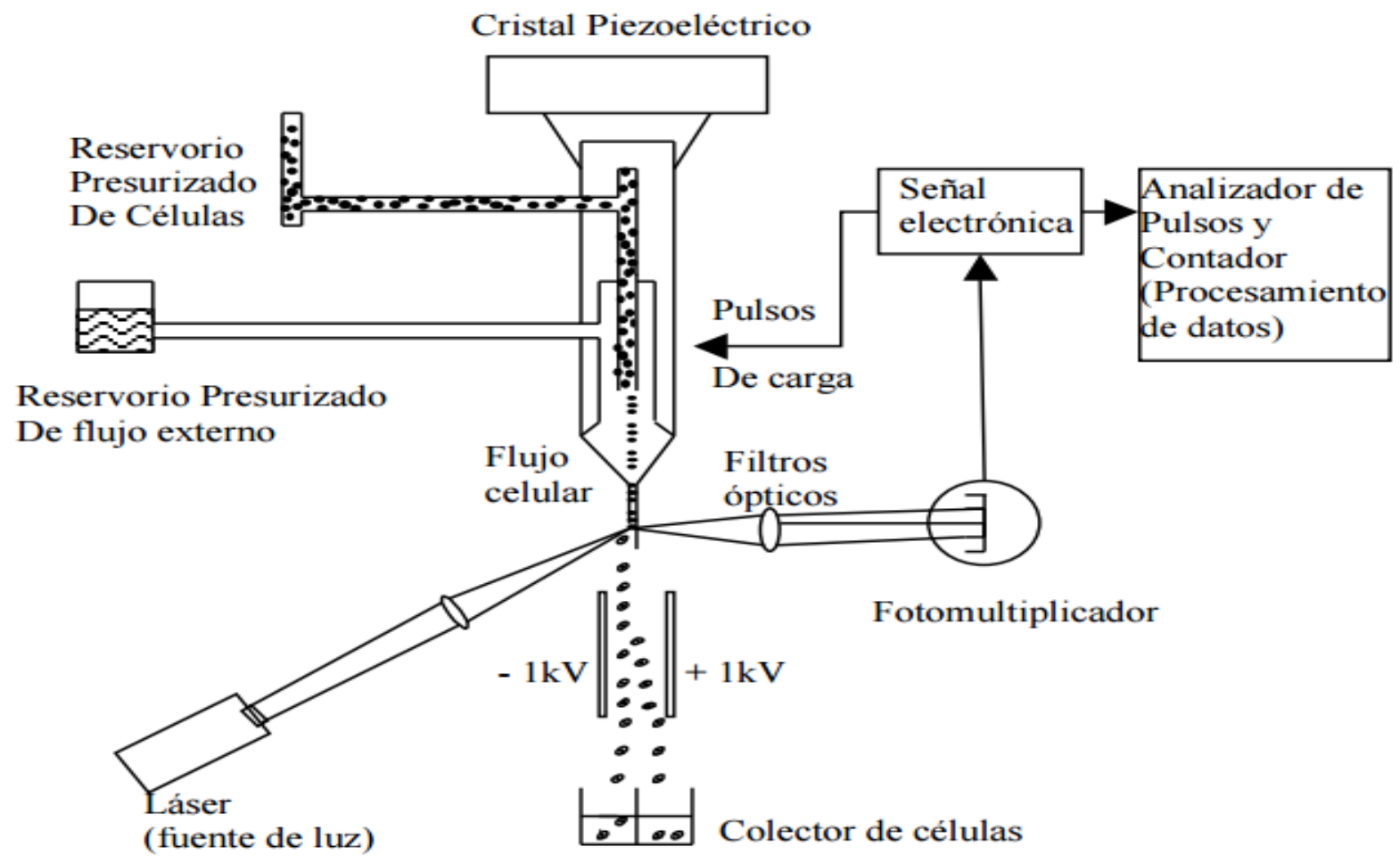

Fig 2. Esquema generalizado de los componentes de un citómetro-sorteador separando y recolectando células. Modificado de: Salgado, 2002.

\section{ÁREAS DE APLICACIÓN DE LA CITOMETRÍA DE FLUJO}

Son innumerables las aplicaciones de la citometría de flujo a la investigación básica, desde campos como la microbiología, hematología, inmunología, citología, patología, biología celular y molecular, entre otros. Por ejemplo la conjugación de marcadores fluorecentes con anticuerpos monoclonales o policlonales ha hecho posible los estudios de la densidad y la distribución de determinantes y receptores de la superficie, y del citoplasma celular, permitiendo identificar subpoblaciones celulares (Barrera et al., 2004). A continuación se pondrán algunas de las aplicaciones que tiene la citometría de flujo en los diferentes campos científicos. 
En el área médica las aplicaciones más relevantes con citometría de flujo se relacionan con la hematología e inmunología clínicas, midiendo parámetros como número y clasificación de células sanguíneas. En la práctica clínica, empezó a usarse en el conteo de linfocitos T en la infección por el virus de inmunodeficiencia humana. En la actualidad esta técnica también es empleada en el conteo de subpoblaciones de linfocitos en pacientes con el virus de la inmunodeficiencia humana así como la caracterización de leucemias agudas y síndromes linfoproliferativos crónicos, entre otros padecimientos. Para la reproducción humana también es una técnica empleada por ser una de las herramientas más objetivas y precisas para analizar la integridad de la cromatina espermática, la integridad del ADN y medir la muerte en una variedad de tipos celulares (Giraldo y González, 2010; Cruz et al., 2010; Barrera et al., 2004).

En la microbiología ambiental presenta nuevas oportunidades para entender la adaptación de los microorganismos a ambientes extremos, nuevos ecosistemas o para realizar la detección rápida de bacterias o virus que son viables pero se encuentran en un estado no cultivable y por lo tanto no detectable por métodos convencionales de cultivo. Si bien muchas estrategias de cultivo redundan en los aislamientos previstos de diferentes fuentes naturales, la gran variedad filogenética y fisiológica de estos aislamientos demuestra la necesidad de desarrollar nuevas estrategias de detección cualitativa. Los análisis por citometría de flujo proveen información sobre el número de células, los tamaños del genoma, la variabilidad de las poblaciones, entre otras características (Laguado, 2007).

Por otra parte en los métodos bioquímicos de análisis celular a citometría de flujo es capaz de proporcionar una información cuantitativa sobre cada célula en particular y permite identificar en una muestra subpoblaciones de células diferentes, incluso cuando están escasamente representadas (Barrera et al., 2004).

En el laboratorio, la citometría de flujo se puede aplicar en la identificación rápida de microorganismos de interés clínico, estudios de susceptibilidad o resistencia a medicamentos, disminución de los tiempos de procesamiento, anualidad y toma de decisiones terapéuticas o la detección temprana de enfermedades infeccionas emergentes y re-emergentes.

El tema ambiental es otro de los campos integrantes donde nuevas aplicaciones de la citometría de flujo se han implementado con éxito. Tal es el caso de la degradación de hidrocarburos, ya que esta degradación se da mayormente por microorganismos en el suelo o agua donde éstos son derramados. Si bien métodos modernos como la Reacción en Cadena de la Polimerasa, el uso de isótopos radiactivos, la determinación de ARN ribosomal consiguen identificar genes que se encuentren involucrados en las vías metabólicas de degradación, estos 
tienen las limitación de ser técnicas costosas, especializadas y de necesitar gran destreza por parte del operador para que el experimento se desarrolle con éxito. En este contexto la citometría de flujo, junto con otras técnicas, permiten la identificación, estudiar la adaptación y observar la evolución de las diferentes comunidades de bacterias involucradas en la degradación de hidrocarburos (Laguado, 2007).

En el campo de la investigación básica aunado a la clínica se utiliza en la cuantificación de ADN, en la expresión fenotípica, en el transporte de fármacos, en la cuantificación de proliferación, la determinación de apoptosis y en el análisis de cascadas de señalización, entre otros (Juárez y Pérez, 2012).

\section{BENEFICIOS EN VETERINARIA}

La citometría surge como una nueva tecnología en los laboratorios clínicos veterinarios al igual que las aplicaciones clínicas en humanos, aunque es bien sabido que la citometría de flujo no se aplica mucho en veterinaria, pero su uso en un futuro aumentará de manera considerable en la medida que sea difundida la posibilidad de su aplicación en otras especies distintas a pequeños roedores. La citometría en veterinaria permite el análisis de una variedad de células animales incluyendo aplicación como el recuento de reticulocitos, recuento de plaquetas, inmunofenotificación de leucemias y linfomas o recuentos diferenciales en medula ósea en felinos, caninos, bovinos y equinos. También se usa para el recuento de subpoblaciones de linfocitos en ovejas, diagnostico veterinario de anemia hemolítica y neoplasias en equinos; en peces se usa para cuantificar y caracterizar algunas poblaciones celulares y estudiar procesos inmunopatológicos; en caso de truchas arcoíris, permite diferenciar básicamente la población de eritrocitos, linfocitos/trombocitos y granulocitos, facilitando el monitoreo de la dinámica de la población celular de manera rápida y confiable. Sin embargo, quizás las aplicaciones más importantes de la citometría en veterinaria se basa en los estudios de la respuesta inmune de infecciones económicamente relevantes para la fabricación de vacunas, el mejoramiento de las vacunas ya existentes, mejoramiento genético de especies bovinas y equinas, el desarrollo de modelos de respuesta inmune en animales como primates no humanos con el fin de adquirir un entendimiento profundo de la patogénesis de ciertas enfermedades infecciosas y la experimentación de vacunas antes de comenzar ensayos en humanos (Alzamora et al., 2015; Laguado, 2007). 
Otra aplicación son las infecciones por Mycobacterium bovis, las cuales revisten implicaciones económicas para los productores de ganado y son una amenaza constante para la salud humana. Al ser parásitos intracelulares que sobreviven y se replican dentro de las células del hospedero su detección por métodos tradicionales es limitada y se requiere una aproximación más avanzada como la biología molecular o la citometría de flujo (Laguado, 2007).

\section{BENEFICIOS EN REPRODUCCIÓN ANIMAL}

En la reproducción animal se usa principalmente en la correlación que existe entre la concentración, viabilidad del esperma y la fertilidad en ganado. Desde los comienzos de la inseminación artificial en bovinos y aves, el método primario para la evaluación de la calidad del semen se basa en la inspección visual de la motilidad del esperma bajo un microscopio con la técnica de contraste de fase. Este método es subjetivo e impreciso dependiendo en gran medida de la experiencia del operador. En la actualidad existen experimentos en los que se han combinado métodos fluorescentes con un citómetro de flujo modificado para el análisis de esperma bovino en estaciones de inseminación artificial. Al usar una versión modificado de un citómetro clínico se ha desarrollado un método rápido, preciso que permite la selección del mejor semen de toros y cerdos. El citómetro de flujo (CF) evalúa la calidad, estructura, función y motilidad del esperma luego de ciclos de congelación/descongelación; valorar daños en el esperma y su calidad para la fertilización (Laguado, 2007).

El estudios de la viabilidad espermática mediante citometría de flujo normalmente se basa en el análisis de la integridad de la membrana plasmática de los espermatozoides, mediante el uso de dos fluorocromos combinados, uno de ellos es capaz de atravesar las membranas plasmáticas dañadas o degeneradas, y por lo tanto permite identificar a las células muertas o en el proceso de degeneración, mientras que el otro es capaz de atravesar membranas celulares intactas y por lo tanto permite identificar la población de células viables. Uno de los fluorocromos más utilizados para esta técnica es el Ioduro de propidio (PI).

Para el análisis de la integridad acrosomal del espermatozoide por CF, es común el uso de una lectina conjugada con un fluorocromo, las lectinas más usadas son las PNA (peanut agglutinin), que se une específicamente a la membrana acrosomal interna de los espermatozoides, y la PSA (pisum sativum agglutinin), la cual se una a la matriz acrosomal y a la membrana acrosomal externa; el uso de estas lectinas permite detectar aquellos espermatozoides que tienen la membrana acrosomal dañada, lo cual posibilita el paso de la lectina, y por lo tanto el fluorógeno, al interior del compartimiento acrosomal. Otra posibilidad 
para identificar la presencia de alteraciones acrosomales es el uso de anticuerpos monoclonales, marcados con un fluorocromo, frente a moléculas especificas del contenido acrosomal (Muiño, 2008).

El uso de lectinas conjugadas con un fluorocromo como la fluorescencia (FITC) o ficoeritrina (PE) permite detectar aquellos espermatozoides que tienen la membrana acrosomal dañada, lo cual posibilita el paso de lectina, y por lo tanto del fluorogeno, al interior del compartimiento acrosomal. Al aplicar el análisis de citometría de flujo, es más común el uso de espermatozoides vivos no permeabilizados, y por tanto la lectina detectara los acrosomas dañados. Otra posibilidad para identificar la presencia de alteraciones acrosomales es el uso de anticuerpos monoclonales, marcados con un fluorocromo, frente a moléculas especificas del contenido acrosomal (Urbina, 2012).

Por otro lado, los parámetros comúnmente evaluados en muestras seminales no son a veces suficientes para descubrir daños en las células. Estos daños producidos y no detectados con los parámetros de rutina, podrían tener repercusión en la fertilidad y/o prolificidad en granja, por ello en los últimos años se han buscado otros marcadores de calidad seminal, como la actividad mitocondrial, el grado de apoptosis, viabilidad de la membrana plasmática, la oxidación espermática, el estado de capacitación y el daño del ADN. Todos estos parámetros son rápidamente y simultáneamente evaluables mediante citometría. Además, se ha demostrado una correlación entre los valores de fragmentación de ADN evaluados y dificultades reproductivas (Ausejo et al., 2016). Otros de los usos que se le está dando a la citometría de flujo para analizar otros aspectos de los espermatozoides es el análisis sobre de la estructura de la cromática espermática, la actividad mitocondrial y su relación con la motilidad (Matas, 1997).

Para el caso del análisis de la funcionalidad mitocondrial con CF, el fluorocromo Rodamina 123 fue el primero que se utilizó para evaluar la funcionalidad mitocondrial de los espermatozoides. Este actúa penetrando las mitocondrias con actividad respiratoria y se acumula en su interior. Al incidir la luz del láser sobre los espermatozoides teñidos, la pieza intermedia de los espermatozoides con mitocondrias activas emite una intensa fluorescencia verde. También existe otra tinción para poder observar la funcionalidad mitocondrial, esta es el fluorocromo JC-1, que se acumula en el interior de las mitocondrias, su actividad respiratoria que es elevada forma agregados intramitocondriales que emiten fluorescencia naranja, y si la actividad respiratoria es baja el JC-1 no llega a formar agregados y emite fluorescencia verde. La Rodamina 123 suele utilizarse en combinación con PI, que tiñe de rojo el núcleo de los 
espermatozoides degenerados y por tanto si actividad mitocondrial. Aunque la Rodamina 123 permite cuantificar la población de espermatozoides con mitocondrias activas, no permite diferenciar el grado de actividad respiratoria de las células (Urbina, 2012; Muiño, 2008).

A partir de la década de 1980 se comenzó a aplicar la citometría de flujo para separar los espermatozoides de acuerdo a sus cromosomas sexuales, realizando así, el sexado de los espermatozoides, separando el contenido cromosómico (ADN) X de aquellos cuyo cromosoma es Y con lo cual podemos predecir de manera segura el sexo de la cría que deseamos obtener al realizar un servicio de Inseminación Artificial (IA) con dosis de semen, cuyos espermatozoides han sido previamente separados; y así usar una muestra que contenga espermatozoides cuyo $\mathrm{ADN}$ sea $\mathrm{X}$ para el caso que se requiera obtener crías hembras y para cuando se requiera disponer de crías machos, se usaría una dosis cuyos espermatozoides contengan ADN Y. El semen al ser evaluado en esta prueba es teñido con el colorante Hoechst 33342 que tiñe al ADN de los espermatozoides y emitir fluorescencia al ser sometido a luz láser. A mayor cantidad de espermatozoides con cromosoma X habrá mayor fluorescencia debido a un mayor porcentaje de $\mathrm{ADN}$ en estos cromosomas comprados a los cromosomas Y (Fig. 3); por ejemplo en el bovino (Bos Taurus y Bos indicus) el análisis citométrico del contenido de ADN indica una

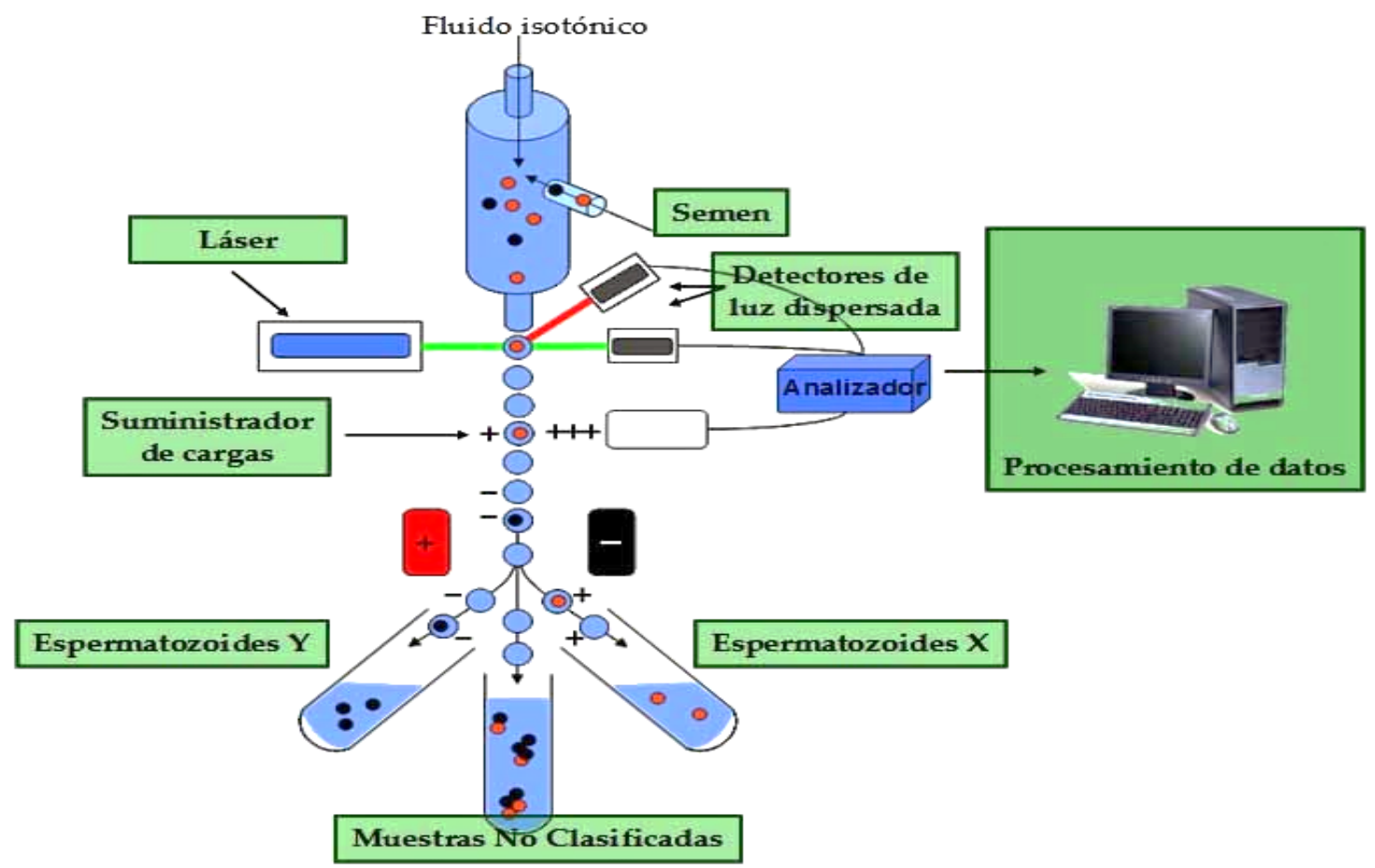

Fig 3. Esquema que muestra la clasificación de los espermatozoides dependiendo la orientación de esperma preferido (eje de flujo en página) en citometría de flujo y como es pasado a su vez a una base de datos en computadora. 
diferencia de aproximadamente de 3,7\% de X sobre la Y. La fluorescencia que produce cada espermatozoide teñido es procesada por un software que permite al operador seleccionar la población espermática con mínima o máxima luminosidad, según el peso que se quiera separar (Ramos, 2013; Urbina, 2012; Villanueva y Mellisho, 2011; Oses et al., 2009).

Los espermatozoides elegidos son cargados eléctricamente, desviados del flujo original en un campo magnético y finalmente recolectados en un tubo para su posterior congelación. Aproximadamente del 100\% de los espermatozoides, un 20\% termina colectado en la fracción X, y un 20\% en la fracción Y; el 60\% restante lo constituyen espermatozoides que no pudieron ser detectados por la técnica, espermatozoides muertos y gotas sin esperma. Adicionalmente se añade otro colorante, yoduro de propidio (PI) que permite determinar los espermatozoides muertos o dañados y de esta manera separarlos de los espermatozoides sexados y viables. Finalmente se somete a un proceso de congelación. El semen sexado se presenta comercialmente congelado, en pajuelas de $0.25 \mathrm{ml}$, las cuales contiene de 1 a 10 millones de espermatozoides. La velocidad de separación que se utiliza actualmente permite obtener 7 pajuelas de 2 millones de espermatozoides X e igual número de Y por hora (figura 4) (Urbina, 2012).

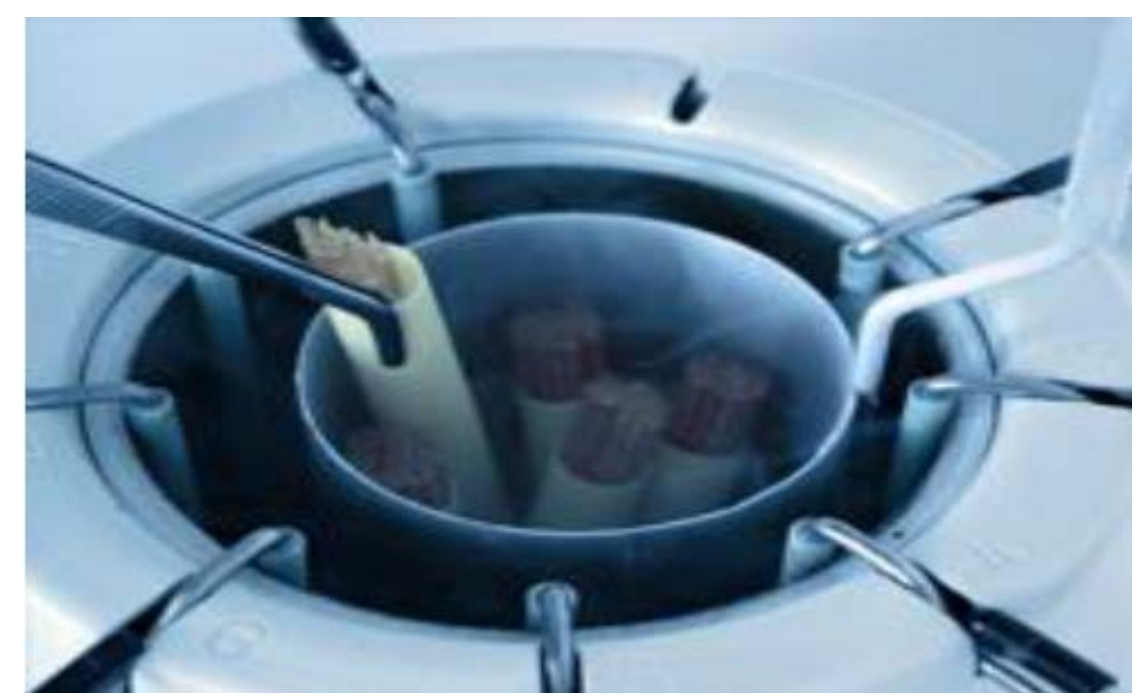

Figura 4. Almacenamiento de pajillas de 0.25 ml. Modificado de: Urbina, 2012.

Para controlar la calidad del semen, se descongela una pajuela por partida y se evalúa la motilidad progresiva y la pureza (proporción del sexo deseado) de las dosis producidas. Las mismas deben tener un mínimo de 35\% de espermatozoides con motilidad progresiva y un 85\% de certeza del sexo para alcanzar los estándares de aprobación (Oses et al., 2009) 
Así a partir de esta técnica se obtiene el denominado semen sexado. Cada dosis de semen sexado contiene en promedio un 90\% de espermatozoides del sexo deseado; además, de que no contiene espermatozoides muertos o dañados y se puede usar para inseminaciones artificiales, fertilización in vitro y transferencia embrionaria (Ramos, 2013; Marini y Galassi, 2011; Oses et al., 2009; Sharpe y Evans 2009).

\section{CONCLUSIONES}

Aunque la citometría de flujo es una técnica que a lo largo del tiempo ha ido evolucionando para convertirse en una forma de análisis celular eficiente en varias disciplinas que requieren valores exactos. En el área de la reproducción animal, actualmente promete ser un método confiable y barato para analizar distintas características espermáticas, contribuyendo de esta manera al mejoramiento reproductivo y genético del ganado, por lo que es necesario seguir investigando al respecto.

\section{BIBLIOGRAFÍA}

Alzamora Gonzales Libertad, De Amat Herbozo Carolina, Colona Vallejos Erasmo, Cervantes Aguilar Elizabeth, Velarde Álvarez Richard Dyer, Aquino Ortega Ronald y Aguilar Luis Miguel Ángel. 2015. Método rápido para la cuantificación de leucocitos sanguíneos y su utilidad en la evaluación del estado de salud en trucha arcoíris Oncorgynchus mykiss. Lat. Am. J. Aquiat. Res. 43 (5): 1019-1023.

Ausejo Raquel, Mendoza Noelia, Miguel Joaquín y Dahmani Yahya. 2016. Calidad seminal: Nuevas tecnologías. Los porcicultores y su entorno 19 (109):149-150.

Barrera Ramírez Lourdes María, Drago Serrano Ma. Elisa, Pérez Ramos Julia, Zamora Ana Cecilia, Gómez Arroyo Fabiola, Sainz Espuñes Teresita del Rosario y Mendoza Pérez Felipe. 2004. Citometría de flujo: vínculo entre la investigación básica y la aplicación clínica. Rev. Inst. Nal. Enf. Resp. Mex. 17 (1): 42-55.

Cruz Ibis, Colmenares Melisa, Berrueta Carrillo Leidith, Gómez Pérez Roald, Montes Henry Berrueta Lisbeth, Salmen Siham y Osuna Jesús Alfonso. 2010. Evaluación de la calidad del espermatozoide humano: comparación entre la integridad del ADN espermático y variables del semen. Invest. Clin. 51 (1): 87-99.

Giraldo Nicolás A. y González John M. 2010. Revisión sistemática en revistas científicas de las publicaciones colombianas con uso de citometría de flujo. Biomédica 30: 32-36. 
Juárez Velázquez Rocío y Pérez Vera Patricia. 2012. Citometría de flujo en la evaluación de enfermedad mínima residual en leucemia linfoblástica aguda. Acta Pediatri. Mex. 33 (4): 198-206.

Laguado José. 2007. Aplicaciones de la citometría de flujo en microbiología, veterinaria y agricultura. Rev. MVZ Córdoba 12 (2): 1077-1095.

Loureiro. 2009. Aplicación de la citometría de flujo en el estudio del genoma vegetal. Ecosistemas 18 (2):103-108.

Marini P.R. y Galassi I. 2011. Relación entre celo-inseminación con semen sexado y porcentaje de preñez en vaquillonas Holstein. Rev. Vet. 22 (1): 52-54.

Matas Parra Carmen. 1997. Análisis mediante citometría de flujo de la respuesta de los espermatozoides de verraco a diferentes medios de incubación. Facultad de Veterinaria. Patología animal. Universidad de Murcia: 16-23.

Muiño Otero Rodrigo. 2008. Evaluación de la motilidad y viabilidad del semen bovino mediante el uso de sistemas casa y citometría de flujo: identificación de subpoblaciones espermáticas. Tesis presentada para la obtención del grado de doctor. Universidad de Santiago de Compostela. Facultad de Veterinaria. Departamento de Patoloxía Animal: 479.

Oses M.V. Teruel M.T. Cabodevila J.A. 2009. Utilización de semen bovino sexado en inseminación artificial, transferencia embrionaria y fertilización in vitro. Rev. Vet. 20 (2): 138-145.

Piedras Josefa. 2006. Citometría de flujo en el diagnóstico y clasificación de padecimientos hematológicos: leucemias agudas, síndromes linfoproliferativos crónicos y glicoproteínas plaquetarias. Revista de Hematología 7 (2): 53-62.

Ramos Coaguila Olger. 2013. Utilización del semen sexado en producción de vacunos. Sistema de Revisiones en Investigación de San Marcos: 1-11.

Salgado Lynn Mílena. 2002. Citometría de flujo: fluorescence-Activated Cell Sortíng (FACS). Curso de Métodos de Biotecnología. Universidad Nacional Autónoma de México. Instituto de Biotecnología: 5.

Sharpe J.C y Evans K.M. 2009. Advances in flow citometry for sperm sexing. Theriogenology 71: 4-10.

Urbina Quito Camilo Ernesto. 2012. Utilización de semen bovino sexado en inseminación artificial, transferencia embrionaria y fertilización in vitro. Monografía de grado, previa a la obtención del título de Médico Veterinario. Universidad de cuenca. Facultad de Ciencias Agropecuarias. Escuela de Medicina Veterinaria y Zootecnia: 40-55. 
Villanueva E. y Mellisho E. 2011. Fertilidad de semen sexado "X” comercial en vacunos de leche. APERMOVA 1(1):106-107. 